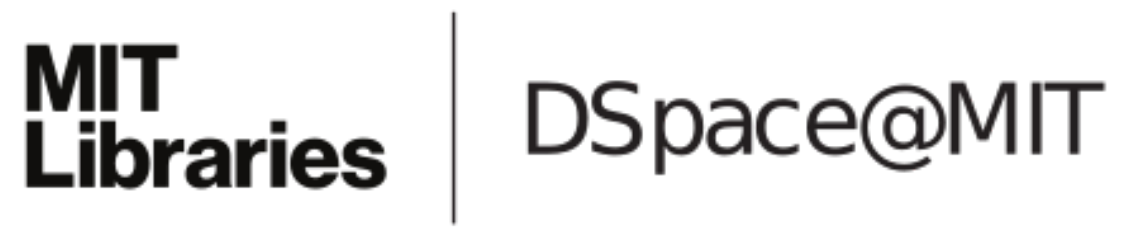

\author{
MIT Open Access Articles
}

Toric-boson model: Toward a topological quantum memory at finite temperature

The MIT Faculty has made this article openly available. Please share how this access benefits you. Your story matters.

Citation: Hamma, Alioscia, Claudio Castelnovo, and Claudio Chamon. "Toric-boson model: Toward a topological quantum memory at finite temperature." Physical Review B 79.24 (2009): 245122. (c) 2009 The American Physical Society.

As Published: http://dx.doi.org/10.1103/PhysRevB.79.245122

Publisher: American Physical Society

Persistent URL: http://hdl.handle.net/1721.1/51820

Version: Final published version: final published article, as it appeared in a journal, conference proceedings, or other formally published context

Terms of Use: Article is made available in accordance with the publisher's policy and may be subject to US copyright law. Please refer to the publisher's site for terms of use. 


\title{
8 \\ Toric-boson model: Toward a topological quantum memory at finite temperature
}

\author{
Alioscia Hamma, ${ }^{1,2}$ Claudio Castelnovo, ${ }^{3}$ and Claudio Chamon ${ }^{4}$ \\ ${ }^{1}$ Perimeter Institute for Theoretical Physics, 31 Caroline Street North, Waterloo, Ontario, Canada N2L $2 Y 5$ \\ ${ }^{2}$ Research Laboratory of Electronics, Massachusetts Institute of Technology, 77 Massachusetts Avenue, Cambridge, \\ Massachusetts 02139, USA \\ ${ }^{3}$ Rudolf Peierls Centre for Theoretical Physics, University of Oxford, 1 Keble Road, Oxford OX1 3NP, United Kingdom \\ ${ }^{4}$ Department of Physics, Boston University, 590 Commonwealth Avenue, Boston, Massachusetts 02215, USA
}

(Received 30 December 2008; revised manuscript received 3 April 2009; published 18 June 2009)

\begin{abstract}
We discuss the existence of stable topological quantum memory at finite temperature. At stake here is the fundamental question of whether it is, in principle, possible to store quantum information for macroscopic times without the intervention from the external world, that is, without error correction. We study the toric code in two dimensions with an additional bosonic field that couples to the defects, in the presence of a generic environment at finite temperature: the toric-boson model. Although the coupling constants for the bare model are not finite in the thermodynamic limit, the model has a finite spectrum. We show that in the topological phase, there is a finite temperature below which open strings are confined and therefore the lifetime of the memory can be made arbitrarily (polynomially) long in system size. The interaction with the bosonic field yields a long-range attractive force between the end points of open strings but leaves closed strings and topological order intact.
\end{abstract}

DOI: $10.1103 /$ PhysRevB.79.245122

PACS number(s): 71.10.Pm, 03.67.Lx, 03.67.Mn, 73.43.Nq

\section{INTRODUCTION}

In recent years, it has become clear that models of computation based on quantum mechanics are radically different from classical ones. ${ }^{1,2}$ However, the quantum wave function of a macroscopic system rapidly decoheres because of the interaction with the environment. ${ }^{3}$ Decoherence is responsible for the appearance of a classical world. ${ }^{4,5}$ Therefore, if we want to process quantum information, we must preserve it from being spoiled by decoherence.

In the past 15 years, a great amount of literature in quantum information has been devoted to the theory of error correction, using several different paradigms..$^{1,2,6}$ We encounter here a profound difference with respect to the classical case: encoding classical information in a system can be robust, without the need for error correction (e.g., magnetic hard drives). On the other hand, the fragility of quantum states to decoherence seems to require quantum error correction. Is that really so? Can one build robust quantum memory at the physical level, just like one does with classical memory, or does quantum mechanics forbid it?

For suitably symmetric models of the environment, there exist decoherence-free subspaces (DFSs). ${ }^{7}$ However, it is controversial whether these subspaces can exist in practice because the conditions to obtain DFSs are unstable. ${ }^{8}$ Moreover, DFSs only exist for a specific model of noise, while we are interested in the question of principle whether a quantum system can be protected from a generic interaction with the environment without operating from the external world (i.e., error correction). The only restriction we shall assume about the way the environment interacts with the system is the locality of the interaction.

If the only requirement is locality of the interaction between the environment and the system, we can imagine encoding information in some collective topological degrees of freedom and obtaining the desired robustness at the physical level. Physical systems that exhibit topological order seem to have the appropriate topological features. ${ }^{9}$ The toric code is an example of a self-correcting quantum memory, that is, a quantum memory that is inherently robust against arbitrary (local) perturbations without need for error correction at zero temperature. . $^{10,11}$

However, robustness against perturbations is not enough. What one needs is a system in which quantum information can be stored for macroscopically long times, in the presence of arbitrary local interactions with the environment at finite temperature $T$. There are not only errors due to virtual processes but, even more importantly, errors due to thermal excitations. For example, the toric code in two and three dimensions (2Ds and 3Ds) is not stable. ${ }^{11-14}$ [See Ref. 15 for a discussion on generic stabilizer codes.] The toric code in four dimensions is stable, ${ }^{11,13,16}$ but interactions in four spatial dimensions are hardly realistic.

One wonders whether quantum information is not only logically different from the classical one but also physically different in that it cannot be stored efficiently in a passive way (in less than four dimensions). Therefore, we are faced with a fundamental question: does a stable quantum memory exist at all or the laws of nature forbid it in three or less dimension?

In this paper, we take a step toward realizing topological quantum memories in two spatial dimensions that are stable against decoherence toward an arbitrary environment. We obtain this result by adding to the toric code a local interaction between the defects and a bosonic field (toric-boson model). This result comes at the cost of a compensating defect energy whose strength diverges with growing system size. However, the protection arising in the toric-boson model is not trivially equivalent to removing thermal defects by introducing an infinite energy cost by itself. In our model, the physical energy scales (namely, the excitation spectrum) remain finite, and it is the induced long-range interactions 
between defects that render the quantum state stable to thermal fluctuations. This is similar to what one usually does in high-energy physics, where infinite coupling constants are used (often needed) to obtain finite-energy spectra.

We show that the toric-boson model realizes a quantum memory that can be stored for macroscopically long times, in the sense that the relaxation times scale (polynomially) with system size. While we fall short of realizing a stable topological quantum memory using local finite coupling terms in the Hamiltonian, our model shows that quantum relaxation times scaling with system size can be obtained in a system with a finite excitation spectrum, using local-coupling terms only.

\section{TORIC CODE}

The toric-code model ${ }^{10}$ is defined on a $2 \mathrm{D} L \times L$ square lattice with periodic boundary conditions and with spin-1/2 degrees of freedom on the $N=2 L^{2}$ edges. On every vertex $s$, we can define the star operator $X_{s}=\prod_{j \in s} \sigma_{j}^{x}$ and on every plaquette $p$ the plaquette operator $Z_{p}=\prod_{j \in p} \sigma_{j}^{z}$. The Hamiltonian of the toric code is

$$
H_{\text {toric }}=-\frac{\Delta}{2}\left(\sum_{s} X_{s}+\sum_{p} Z_{p}\right) .
$$

The operators $X_{s}, Z_{p}$ all commute and therefore their eigenvalues are good quantum numbers for labeling the states. Nevertheless, they are not all independent because periodic boundary conditions imply $\Pi_{s} X_{s}=\Pi_{p} Z_{p}=1$. The two degrees of freedom not labeled by the above quantum numbers are the two topological qubits defined by the algebra of the Pauli operators $\left\{X_{L_{1}}=\prod_{j \in L_{1}} \sigma_{j}^{x}, \quad Z_{M_{2}}=\prod_{j \in M_{2}} \sigma_{j}^{z},\right\}$ and $\left\{X_{L_{2}}, Z_{M_{1}}\right\}$ defined similarly, where $L_{1}, L_{2}$ and $M_{1}, M_{2}$ are appropriately defined noncontractible loops around the torus.

Two topological qubits can then be defined as the subspaces on which the two Pauli algebras $\left(Z_{M_{1}}, X_{L_{2}}\right)$ and $\left(Z_{M_{2}}, X_{L_{1}}\right)$ act, respectively. For example, one can choose the two basis states for the first qubit to be the eigenvectors $|0\rangle$ and $|1\rangle$ of $Z_{M_{1}}$ with eigenvalues +1 and -1 . In this case, the operator $X_{L_{2}}$ flips the qubit $\left(X_{L_{2}}|0\rangle=|1\rangle\right)$ while the operator $Z_{M_{2}}$ can be used to change the phase of a qubit state [e.g., $\left.Z_{M_{2}}(|0\rangle+|1\rangle) / \sqrt{2}=(|0\rangle-|1\rangle) / \sqrt{2}\right]$.

Since the model has a spectral gap $\Delta$, the information encoded in the topological qubits is stable against arbitrary local perturbations. ${ }^{10}$ However, it is fragile when interacting with an arbitrary environment at finite temperature (even if it is local). ${ }^{11-14} \mathrm{~A}$ heuristic argument for the fragility can be formulated as follows. Consider for concreteness $H_{\mathrm{I}}$ $=g \sum_{j=1}^{N}\left(\sigma_{j}^{x} \otimes f_{j}+\sigma_{j}^{z} \otimes f_{j}\right)$ to be the interaction Hamiltonian between the system and the environment. This interaction will create pairs of defects (anyons) with probability $P \sim \exp ($ $\left.-\Delta / k_{B} T\right)$ and move them about in a Brownian motion. When two anyons travel around the noncontractible holes of the torus to recombine and annihilate, a logical error is produced, namely, a noncontractible loop. [For example, a pair of $Z_{p}$ defects (generated by $\sigma^{x}$ operators) that travel around a loop of type $L_{2}$ will change the eigenvalue of $Z_{M_{1}}$ and $\forall M_{1}$.] Since the anyons propagate freely, the time scale for an iso- lated pair to induce an error is proportional to $L^{2}$. However, the very same noninteracting nature that ensures free motion of the defects causes a nonvanishing density of them at any given temperature. Therefore, one can envisage faster collective processes whereby pairs stretch only as far as reaching anyons from neighboring pairs and by annihilating them, they lead to longer overall propagation paths. The time scale to induce an error via these processes is roughly given by the time it takes for a pair to stretch over a distance equal to the average interparticle separation $t_{\text {rel }} \sim \exp \left(\Delta / k_{B} T\right)$. The relaxation time is therefore microscopic because it does not scale with system size.

The reason why end points of strings (the anyons) can propagate freely is that here only the boundary of stringlike objects pays an energy and a longer string does not have a larger boundary. So one way of confining these objects is to go to higher dimensions. In the 3D toric code, topological information is stored in noncontractible membranes and winding loops. Defects to these operators acquire the form of open strings and open membranes, respectively. While open strings still behave as in $2 \mathrm{D}$, with end points propagating freely, an open membrane costs energy that grows with the size of its boundary (a closed string), and it is therefore confined. Since only one of the two operators is preserved at finite temperature, the 3D toric code can only encode classical information. ${ }^{13,14}$ In four dimensions, true quantum memory can be stored for times that are exponentially large in $L$ because both types of errors are membranes. ${ }^{11,13,16}$

One can think of confining the strings by adding a tension term to the Hamiltonian. There is a critical value of the coupling with this field such that the open strings are confined. ${ }^{17}$ Unfortunately, the tension term does not distinguish between open and closed strings. If open strings are confined, the system undergoes a quantum phase transition where topological order is destroyed and with it the degenerate ground state encoding the qubit is also lost. In fact, the phase in which the strings are confined can be mapped to the ferromagnetic phase of the $2 \mathrm{D}$ Ising model $^{18}$ and we can only encode classical information in such system.

\section{TORIC-BOSON MODEL}

If we want to hinder the propagation of the anyons without affecting the loops and spoiling topological order, we must find an interaction that only couples the end points of strings. A projector onto the subspace of open strings would allow us to give a tension only to open strings. But this term would be highly nonlocal and therefore not realistic. Another possibility is to add a long-range force that makes the anyons attract, as it was suggested in Ref. 11. However, the problem is whether the needed attractive force can materialize from a purely local Hamiltonian.

As we show in the following, one can reach this goal with a local Hamiltonian by coupling the $2 \mathrm{D}$ toric code to bosonic fields, which will have the desired confining effect in the presence of a completely generic bath acting on both systems. However, we shall achieve this at the cost of a compensating energy term in the Hamiltonian that scales with the size of the system. The bosonic fields will affect open strings 
by attracting the end points, while they have no effect whatsoever on closed strings. In this way, they protect the topological qubit while preserving topological order.

Let $n_{s}$ and $n_{p}$ be the defect-density operators [namely, $n_{s}$ $=\left(1-X_{s}\right) / 2$ and $n_{p}=\left(1-Z_{p}\right) / 2$, respectively] taking values 0 and 1 on the stars/plaquettes of a square lattice (with spacing set to unity). This density will couple locally to the displacement field defined in the continuum. We consider the case of elastic waves (phonons) on the surface of the torus, two in plane and one out of plane. These elastic deformations are denoted by $\varphi(\mathbf{x}, t), \vec{\phi}(\mathbf{x}, t)=\left(\phi^{x}(\mathbf{x}, t), \phi^{y}(\mathbf{x}, t)\right)$, where $\mathbf{x}$ takes values in the continuum [with centers of plaquettes labeled by $\mathbf{x}_{p}$ and those of stars by $\left.\mathbf{x}_{s}\right]$.

The densities $n_{s}$ and $n_{p}$ couple to the out-of-plane phonons piezoelectrically $(\varphi)$ (Ref. 19) and to the in-plane phonons in a rotationally invariant form (via $\left.\partial_{x} \phi^{x}+\partial_{y} \phi^{y}\right)$. In the case of piezoelectric coupling, one has to take proper care of the center-of-mass (CM) mode. One can work with $\varphi_{\mathbf{k}=0}$ pinned to the origin (basically, the entire mass of the crystal behaves as a large classical object). This is physical since what matters in the piezocoupling are the elastic deformations and uniform translations should not affect the system; by working with coordinates relative to the CM, this is ensured.

One can expand $\varphi(\mathbf{x}, t), \quad \phi^{i}(\mathbf{x}, t)$ using creation and annihilation operators $a_{\mathbf{k}}, a_{\mathbf{k}}^{\dagger}, b_{\mathbf{k}}^{(i)}, b_{\mathbf{k}}^{(i) \dagger}$ satisfying the harmonic-oscillator algebra as

$$
\varphi(\mathbf{x}, t)=\frac{1}{\sqrt{V}} \sum_{\mathbf{k} \neq 0} \frac{1}{\sqrt{2 \omega_{\mathbf{k}}}}\left[a_{\mathbf{k}}(t) e^{i \mathbf{k} \cdot \mathbf{x}}+a_{\mathbf{k}}^{\dagger}(t) e^{-i \mathbf{k} \cdot \mathbf{x}}\right]
$$

and

$$
\phi^{i}(\mathbf{x}, t)=\frac{1}{\sqrt{V}} \sum_{\mathbf{k} \neq 0} \frac{1}{\sqrt{2 \Omega_{\mathbf{k}}}}\left[b_{\mathbf{k}}^{(i)}(t) e^{i \mathbf{k} \cdot \mathbf{x}}+b_{\mathbf{k}}^{(i) \dagger}(t) e^{-i \mathbf{k} \cdot \mathbf{x}}\right],
$$

with $i=x, y$, thus implying the canonical equal-time commutation relations

$$
\begin{gathered}
{\left[\phi^{i}(\mathbf{x}, t), \dot{\phi}^{i}\left(\mathbf{x}^{\prime}, t\right)\right]=i \delta\left(\mathbf{x}-\mathbf{x}^{\prime}\right),} \\
{\left[\varphi(\mathbf{x}, t), \dot{\varphi}\left(\mathbf{x}^{\prime}, t\right)\right]=i \delta\left(\mathbf{x}-\mathbf{x}^{\prime}\right)}
\end{gathered}
$$

for the displacement fields. The Hamiltonian for the fields is given by

$$
H_{\text {boson }}=\sum_{\mathbf{k} \neq 0} \omega_{\mathbf{k}} a_{\mathbf{k}}^{\dagger} a_{\mathbf{k}}+\sum_{\mathbf{k} \neq 0, i=x, y} \Omega_{\mathbf{k}} b_{\mathbf{k}}^{(i) \dagger} b_{\mathbf{k}}^{(i)} .
$$

The interaction Hamiltonian reads as

$$
H_{\mathrm{int}}=\sum_{\ell=s, p} n_{\ell}\left\{g_{\omega} \varphi\left(\mathbf{x}_{\ell}\right)+g_{\Omega}\left[\partial_{x} \phi^{x}\left(\mathbf{x}_{\ell}\right)+\partial_{y} \phi^{y}\left(\mathbf{x}_{\ell}\right)\right]\right\}
$$

(notice that $g_{\omega}$ and $g_{\Omega}$ have different dimensions) and the toric-boson Hamiltonian can thus be written as

$$
H=H_{\text {toric }}+H_{\text {boson }}+H_{\text {int }} \text {. }
$$

After substituting Eqs. (2) and (3) into the interaction Hamiltonian (6) and introducing new creation and annihilation operators

$$
\begin{gathered}
\alpha_{\mathbf{k}} \equiv a_{\mathbf{k}}+\frac{g_{\omega} \widetilde{\boldsymbol{\rho}}^{\dagger}(\mathbf{k})}{\sqrt{2 V} \omega_{\mathbf{k}}^{3 / 2}}, \\
\beta_{\mathbf{k}}^{(i)} \equiv b_{\mathbf{k}}^{(i)}+\frac{i g_{\Omega} \mathbf{k}_{i} \widetilde{\boldsymbol{\rho}}^{\dagger}(\mathbf{k})}{\sqrt{2 V} \Omega_{\mathbf{k}}^{3 / 2}} \quad(i=x, y),
\end{gathered}
$$

the total Hamiltonian $H$ of the system can be written as

$$
\begin{aligned}
H= & H_{\text {toric }}+\sum_{\mathbf{k} \neq 0} \omega_{\mathbf{k}} \alpha_{\mathbf{k}}^{\dagger} \alpha_{\mathbf{k}}+\sum_{\mathbf{k} \neq 0, i=x, y} \Omega_{\mathbf{k}} \beta_{\mathbf{k}}^{(i) \dagger} \beta_{\mathbf{k}}^{(i)} \\
& -\frac{1}{V} \sum_{\mathbf{k} \neq 0}|\widetilde{\rho}(\mathbf{k})|^{2}\left(\frac{g_{\omega}^{2}}{2 \omega_{\mathbf{k}}^{2}}+\frac{g_{\Omega}^{2}\left(k_{x}^{2}+k_{y}^{2}\right)}{2 \Omega_{\mathbf{k}}^{2}}\right),
\end{aligned}
$$

where $\tilde{\rho}(\mathbf{k})=\sum_{\ell} n_{\ell} e^{i \mathbf{k} \cdot \mathbf{x}_{\ell}}$ is the Fourier transform of $\rho(x)$ $=\Sigma_{\ell} n_{\ell} \delta\left(\mathbf{x}-\mathbf{x}_{\ell}\right)$. Here we have assumed that the defects are smeared over the corresponding plaquette/star so as to regularize $\delta(\mathbf{x})$ and remove ultraviolet divergences.

Notice that the first two terms in Eq. (10) lead to precisely the same energy levels as those of the bosonic modes in the absence of the defects [Eq. (5)]. This holds true even for thermal averages in mixed states. Therefore, the phononic fields added to the toric-code Hamiltonian give rise to an effective interaction potential $V_{d}$ given by the last term in Eq. (10), irrespective of temperature. Notice that $V_{d}$ is always negative, independent of the arrangement of the defects, and thus it favors defect proliferation, which will need to be appropriately counter balanced later on.

Consider the case of $\mathcal{N} \equiv \mathcal{N}_{X}+\mathcal{N}_{Z}$ defect pairs, at positions $\mathbf{x}_{a}$, with $\ell=1, \ldots, 2 \mathcal{N}_{X}$ labeling the sites with star-type defects and $\ell=2 \mathcal{N}_{X}+1, \ldots, 2 \mathcal{N}$ labeling the sites with plaquette-type defects. The effective interaction potential $V_{d}$ can then be written as

$$
\begin{aligned}
V_{d}\left(\mathbf{x}_{1}, \ldots, \mathbf{x}_{2 \mathcal{N}}\right)= & -\sum_{\ell, \ell^{\prime}=1}^{2 \mathcal{N}} n_{\ell} n_{\ell^{\prime}} \frac{1}{V} \\
& \times \sum_{\mathbf{k} \neq 0}\left[\frac{g_{\omega}^{2}}{2 \omega_{\mathbf{k}}^{2}}+\frac{g_{\Omega}^{2}|\mathbf{k}|^{2}}{2 \Omega_{\mathbf{k}}^{2}}\right] e^{i \mathbf{k} \cdot\left(\mathbf{x}_{\ell}-\mathbf{x}_{\ell^{\prime}}\right)} \\
= & \frac{1}{2} \sum_{\ell, \ell^{\prime}=1}^{2 \mathcal{N}} n_{\ell} n_{\ell^{\prime}}\left[V_{\omega}\left(\mathbf{x}_{\ell}-\mathbf{x}_{\ell^{\prime}}\right)+V_{\Omega}\left(\mathbf{x}_{\ell}-\mathbf{x}_{\ell^{\prime}}\right)\right],
\end{aligned}
$$

where

$$
\begin{gathered}
V_{\omega}\left(\mathbf{x}_{\ell}-\mathbf{x}_{\ell^{\prime}}\right) \equiv-\frac{1}{V} \sum_{\mathbf{k} \neq 0} \frac{g_{\omega}^{2}}{\omega_{\mathbf{k}}^{2}} e^{i \mathbf{k} \cdot\left(\mathbf{x}_{\ell^{-}} \mathbf{x}_{\ell^{\prime}}\right)}, \\
V_{\Omega}\left(\mathbf{x}_{\ell}-\mathbf{x}_{\ell^{\prime}}\right) \equiv-\frac{1}{V} \sum_{\mathbf{k} \neq 0} \frac{g_{\Omega}^{2}|\mathbf{k}|^{2}}{\Omega_{\mathbf{k}}^{2}} e^{i \mathbf{k} \cdot\left(\mathbf{x}_{\ell^{-}} \mathbf{x}_{\ell^{\prime}}\right)} .
\end{gathered}
$$

For acoustic phonons with dispersion $\omega_{\mathbf{k}}=v_{\omega}|\mathbf{k}|$ and $\Omega_{\mathbf{k}}$ $=v_{\Omega}|\mathbf{k}|$, the potential $V_{\omega}(\mathbf{r})$ becomes Coulombic in $d$ dimensions, 


$$
\nabla^{2} V_{\omega}(\mathbf{r})=\frac{g_{\omega}^{2}}{v_{\omega}^{2}} \frac{1}{V} \sum_{\mathbf{k} \neq 0} e^{i \mathbf{k} \cdot \mathbf{r}}=\frac{g_{\omega}^{2}}{v_{\omega}^{2}}\left[\delta^{(d)}(\mathbf{r})-\frac{1}{V}\right] .
$$

Namely, the potential generated by a pointlike charge in a uniform compensating background distribution is

$$
V_{\omega}(\mathbf{r})= \begin{cases}\frac{g_{\omega}^{2}}{v_{\omega}^{2}} \frac{\Gamma(d / 2)}{2 \pi^{d / 2}} \frac{1}{2-d}|\mathbf{r}|^{2-d}+C & d \neq 2 \\ \frac{g_{\omega}^{2}}{v_{\omega}^{2}} \frac{1}{2 \pi} \ln |\mathbf{r}|+C & d=2,\end{cases}
$$

where the uniform compensating background is irrelevant due to the absence of the $\mathbf{k}=0$ term in Eq. (12). For the same reason, $\int V_{\omega}(\mathbf{x}) d^{d} x=0$, which fixes the constant $C \equiv$ $-g_{\omega}^{2} /\left(2 \pi v_{\omega}^{2}\right) \ln \xi_{L}$ with $\xi_{L}$ on the order of the system size $L$ in two dimensions (the actual value will depend on details about the shape of the system).

For $g_{\omega}>0$, the potential $V_{\omega}(\mathbf{r})$ acts therefore as a "gravitational" potential with Newton constant $G$ $=\left(2 v_{\omega}^{2} \pi^{d / 2}\right)^{-1} \Gamma(d / 2)$, attracting particles of mass $g_{\omega}$.

The in-plane phonon contribution amounts to

$$
V_{\Omega}(\mathbf{r})=-\frac{g_{\Omega}^{2}}{v_{\Omega}^{2}} \frac{1}{V} \sum_{\mathbf{k} \neq 0} e^{i \mathbf{k} \cdot \mathbf{r}}=-\frac{g_{\Omega}^{2}}{v_{\Omega}^{2}}\left[\delta^{(d)}(\mathbf{r})-\frac{1}{V}\right]
$$

and we can finally combine the two contributions into

$$
\begin{aligned}
V_{\Omega}(\mathbf{r})+V_{\omega}(\mathbf{r}) & =\frac{g_{\omega}^{2}}{v_{\omega}^{2}} \frac{1}{2 \pi} \ln \frac{|\mathbf{r}|}{a} \\
& +\left[\frac{g_{\Omega}^{2}}{v_{\Omega}^{2}} \frac{1}{V}-\frac{g_{\omega}^{2}}{v_{\omega}^{2}} \frac{1}{2 \pi} \ln \frac{\xi_{L}}{a}\right] \\
& -\frac{g_{\Omega}^{2}}{v_{\Omega}^{2}} \delta^{(2)}(\mathbf{r}),
\end{aligned}
$$

where we separated for convenience $\ln \left(|\mathbf{r}| / \xi_{L}\right)$ into $\ln (|\mathbf{r}| / a)-\ln \left(\xi_{L} / a\right)$ (a being the lattice constant, $|\mathbf{r}| / a>1$, $\forall \mathbf{r}$ ). The first term is the desired confining interaction (which we discuss below) while terms (16b) and (16c) are byproducts that need to be appropriately taken care of. By tuning the ratio between $g_{\omega} / v_{\omega}$ and $g_{\Omega} / v_{\Omega}$, one can obtain that the two parts of Eq. (16b) cancel each other out exactly, at the cost however of generating a system-size scaling coupling constant in Eq. (16c)

$$
\frac{g_{\Omega}^{2}}{v_{\Omega}^{2}}=\frac{g_{\omega}^{2}}{v_{\omega}^{2}} \frac{V}{2 \pi} \ln \frac{\xi_{L}}{a} \sim L^{2} \ln L .
$$

Therefore, the phonon-mediated effective interaction between the defects can be written as

$$
\begin{aligned}
V_{d}\left(\mathbf{x}_{1}, \ldots, \mathbf{x}_{2 \mathcal{N}}\right)= & \frac{g_{\omega}^{2}}{v_{\omega}^{2}} \frac{1}{4 \pi} \sum_{\ell, \ell^{\prime}=1}^{2 \mathcal{N}} n_{\ell} n_{\ell^{\prime}} \ln \frac{\left|\mathbf{x}_{\ell}-\mathbf{x}_{\ell^{\prime}}\right|}{a} \\
& -\frac{g_{\omega}^{2}}{v_{\omega}^{2}} \frac{1}{4 \pi}\left(V \ln \frac{\xi_{L}}{a}\right) \sum_{\ell, \ell^{\prime}=1}^{2 \mathcal{N}} n_{\ell} n_{\ell^{\prime}} \delta^{(2)}\left(\mathbf{x}_{\ell}-\mathbf{x}_{\ell^{\prime}}\right),
\end{aligned}
$$

$$
\begin{aligned}
= & \frac{g_{\omega}^{2}}{v_{\omega}^{2}} \frac{1}{4 \pi} \sum_{\ell, \ell^{\prime}=1}^{2 \mathcal{N}} n_{\ell} n_{\ell^{\prime}} \ln \frac{\left|\mathbf{x}_{\ell}-\mathbf{x}_{\ell^{\prime}}\right|}{a} \\
& -\frac{g_{\omega}^{2}}{v_{\omega}^{2}} \frac{1}{4 \pi}\left(V \ln \frac{\xi_{L}}{a}\right) 2 \mathcal{N} \delta^{(2)}(0),
\end{aligned}
$$

where the $\delta$ function has been regularized at short distances just like one does for the Coulomb potential.

The remaining unwanted term-coming from Eq. (16c) plays the role of a chemical potential that favors thermal defects and whose strength scales with the size of the system. In order to preserve the fact that the defect density vanishes at zero temperature, we must neutralize this contribution by adding a compensating energy cost for the defects that also grows with system size.

Since this unwanted term scales linearly in the number of defects $\mathcal{N}$, it can be removed by the further addition of a chemical potential to the Hamiltonian of the system. Indeed, the role of the in-plane phonons in our model is to prevent the arising of undesired defect-favoring long-range interactions that scale with the square of the number of defects $\mathcal{N}^{2}$ and could not be removed by the addition of a local onebody term in the Hamiltonian.

In conclusion, we obtain a purely gravitational defect interaction of the form

$$
V_{d}\left(\mathbf{x}_{1}, \ldots, \mathbf{x}_{2 \mathcal{N}}\right)=\frac{g_{\omega}^{2}}{v_{\omega}^{2}} \frac{1}{4 \pi} \sum_{\ell, \ell^{\prime}=1}^{2 \mathcal{N}} n_{\ell} n_{\ell^{\prime}} \ln \frac{\left|\mathbf{x}_{\ell}-\mathbf{x}_{\ell^{\prime}}\right|}{a}
$$

An important comment is in order. While we did add an infinite $\left(\sim L^{2} \ln L\right)$ coupling term to the Hamiltonian in order to arrive at the effective interaction shown above, this is not equivalent to trivially introducing an infinite energy cost for the defects, which would forbid their presence altogether. In fact, the energy to add a pair of defects in our model remains finite (the environment acts locally on the system and therefore defects are always produced close to each other and move one step at a time).

The situation is similar in high energy, where the bare Hamiltonian often has diverging coupling constants but it carries nonetheless physical meaning as the spectral gaps are finite.

\section{A. Robustness of quantum memory}

Once we obtain an effective long-range gravitational attraction between the defects, we can argue for the stability of the quantum memory. It is well known that the partition function of $\mathcal{N}$ particles of mass $m$ interacting via a 2D gravitational potential $u(r)=G m^{2} \ln (r)$ becomes divergent below a finite temperature $T_{*}=\mathcal{N} G m^{2} / 4$ (see e.g., Ref. 20). The gravitational forces overcome the entropic contribution and lead to a collapse, where all the particles coalesce to a single point. In the toric-boson model, where the massive particles correspond to the defective stars and plaquettes, their number is controlled by the chemical potential due to the ratio $\Delta / T$. Therefore, given that the number of particles is topologically 
constrained to be even, the equilibrium density of defects vanishes below the finite temperature $T_{*}(\mathcal{N}=2)=G m^{2} / 2$.

In this temperature regime, thermal processes connecting two different topological sectors are essentially limited to creation, diffusion, and annihilation of individual pairs of particles. Such processes must overcome the energy barrier of taking one particle around the whole system, which scales with the logarithm of the system size, thus yielding a characteristic macroscopic time scale $t_{\mathrm{rel}} \sim \exp [\alpha \ln (L) / T]$ $\sim L^{\alpha / T}$ which is polynomial in system size $L[\operatorname{poly}(L)]$.

\section{ROBUSTNESS OF THE TORIC-BOSON MODEL}

An essential ingredient to obtain the effective gravitational potential between the defects lies in the gaplessness of the phonon modes involved in the system. In order to ensure robustness against thermal fluctuations in the toric-boson model, one should therefore also ensure that the action of the environment on the phonon modes does not open a gap (e.g., via introducing effective interactions between the different bosonic modes).

In our case, the gaplessness $\left(\omega_{\mathbf{k}} \sim|\mathbf{k}|\right)$ of phononic excitations is protected by translational symmetry. Even if the elastic medium is inhomogeneous, it satisfies the equation of motion $m(\mathbf{x}) \ddot{\phi}(\mathbf{x}, t)=\int d^{d} y K(\mathbf{x}, \mathbf{y}) \phi(\mathbf{y}, t)$, where the condition $\int d^{d} y K(\mathbf{x}, \mathbf{y})=0$ follows from uniform translations $\phi(\mathbf{x}, t)$ = const; this condition on the $K(\mathbf{x}, \mathbf{y})$ is similar to that in a master equation for stochastic systems ${ }^{21}$ with the difference that the wave equation is second order. It thus follows from generic arguments on diffusion in inhomogeneous media that the dispersion of the phonons for long wavelengths must be linear, $\omega_{\mathbf{k}} \sim|\mathbf{k}|$. Notice that this conclusion holds even if the inhomogeneous elastic medium breaks translation symmetry [in the form of an inhomogeneous $m(\mathbf{x})$ and $K(\mathbf{x}, \mathbf{y})]$. The key point is that at long distances, acoustic sound waves propagate in the effective medium. Notice also that to attempt to pin the displacement fields with a rigid substrate potential is an illusion, as one must consider the elastic properties of the substrate itself. The linearly dispersing acoustic modes in the effective medium at large distances are responsible for the long-range behavior of $v(\mathbf{r})$, which remains unchanged after tracing out the environment. [One can bypass the issue of the linear dispersion and compute directly $V_{\omega}(\mathbf{r})$ in the inhomogeneous medium: $V_{\Omega}\left(\mathbf{x}^{\prime}, \mathbf{x}\right)=\int_{0}^{\infty} d t P_{W}\left(\mathbf{x}^{\prime}, \mathbf{x} ; t\right)$, where $P_{W}\left(\mathbf{x}^{\prime}, \mathbf{x} ; t\right)$ is the probability to diffuse from $\mathbf{x}$ to $\mathbf{x}^{\prime}$ in time $t$, computed using the transition rate $W(\mathbf{x}, \mathbf{y})$ $=K(\mathbf{x}, \mathbf{y}) / m(\mathbf{x})$.]

\section{CONCLUSIONS AND OUTLOOK}

In this paper, we have shown how a system with topological order like the toric code can be made robust to local interactions with a generic environment below a finite temperature $T_{*}$, if the defects in the system are coupled to a phononic field. The defects feel a long-range attractive force that prevents them from moving freely around the torus. The long-range force is not spoiled by the interaction of the bosons with the environment as long as the dispersion relation is preserved. In our model, phonons are protected by translational symmetry and the dispersion relation yields a logarithmic potential resulting in a relaxation-time scaling as poly $(L)$. Therefore, quantum information can be stored for arbitrarily long times, scaling with the size of the system.

In the model described here, we need to introduce compensating terms that scale as $L^{2} \ln L, L$ being the linear size of the system and thus diverge in the thermodynamic limit. However, we do so while preserving the finite nature of the low-energy spectrum of the system. We believe that this is a step farther with respect to the simple addition of an infinite energy cost for the defects in the Hamiltonian. Our model shows that, in principle, quantum mechanics allows for the preservation of quantum information for arbitrarily long times, scaling with the size of the system.

There are a number of open problems left to investigate. First and foremost, can one construct a model with similar physics where all the interactions are not only local but also finite?

Second, for reliable quantum topological memory, poly $(L)$ is not ideal. It takes in general polynomial time to operate on a topological qubit (e.g., the readout at finite temperature $\left.{ }^{14}\right)$. Our system would be viable only in the lowtemperature limit, where the polynomial relaxation-time scale $t_{\text {rel }} \sim L^{\alpha / T}$ is much larger than the (temperature independent) polynomial operational time scale. A stronger quantum memory could be realized in a system that exhibits exponential relaxation-time scales $t_{\text {rel }} \sim \exp (L)$. This could be obtained using a bosonic field with protected gapless excitations with dispersion $\omega \sim|k|^{3}$. There are other topologically ordered systems with the right cubic dispersion relation, whose gaplessness is protected by topological order itself, resulting in a very strong protection. ${ }^{22}$ Therefore, a toric code interacting with the appropriate system would feature exponentially large relaxation times. We think that the robustness of quantum memory in such system is the true meaning of the full survival of quantum topological order at finite temperature.

\section{ACKNOWLEDGMENTS}

We acknowledge important discussions with M. Hastings, X.-G. Wen, and D. Lidar. We are particularly grateful to M. Hastings for pointing out some of the undesired terms that arise in our Hamiltonian. Such terms would have led to phase-separation effects in the system and we had missed them in a previous version of the manuscript. Research at Perimeter Institute for Theoretical Physics is supported in part by the Government of Canada through NSERC and by the Province of Ontario through MRI. This work was supported in part by EPSRC under Grant No. GR/R83712/01 (C.C.) and by a grant from the Foundational Questions Institute (fqxi.org), a grant from xQIT at MIT (A.H.). A.H. and C.C. thank the BU Condensed Matter Theory visitors program for its hospitality. 
${ }^{1}$ D. Deutsch, Proc. R. Soc. London, Ser. A 400, 97 (1985); Science 270, 255 (1995); A. Ekert and R. Josza, Rev. Mod. Phys. 68, 733 (1996).

${ }^{2}$ M. Nielsen and I. L. Chuang, Quantum Computation and Quantum Information (Cambridge University Press, Cambridge, England, 2000).

${ }^{3}$ I. L. Chuang, R. Laflamme, P. W. Shor, andW. H. Zurek, Science 270, 1633 (1995).

${ }^{4}$ W. H. Zurek, Phys. Today 44(10), 36 (1991); W. H. Zurek, S. Habib, and J. P. Paz, Phys. Rev. Lett. 70, 1187 (1993); W. Zurek, Phys. Rev. D 26, 1862 (1982).

${ }^{5}$ D. Giulini, E. Joos, C. Kiefer, J. Kupsch, I. O. Stamatescu, and H. D. Zeh, Decoherence and the Appearance of the Classical World (Springer-Verlag, Berlin, 1996).

${ }^{6}$ P. W. Shor, Phys. Rev. A 52, R2493 (1995); A. M. Steane, Phys. Rev. Lett. 77, 793 (1996); E. Knill, R. Laflamme, and W. H. Zurek, Proc. R. Soc. London, Ser. A 454, 365 (1998); D. Gottesman, Ph.D. thesis, California Institute of Technology, 1997.

${ }^{7}$ P. Zanardi and M. Rasetti, Phys. Rev. Lett. 79, 3306 (1997); D. A. Lidar, I. L. Chuang, and K. B. Whaley, ibid. 81, 2594 (1998).

${ }^{8}$ R. Alicki, Physica A 150, 455 (1988).

${ }^{9}$ X. G. Wen, Phys. Rev. B 40, 7387 (1989); X. G. Wen and Q. Niu, ibid. 41, 9377 (1990); X.-G. Wen, Int. J. Mod. Phys. B 4, 239 (1990); Adv. Phys. 44, 405 (1995).

${ }^{10}$ A. Kitaev, Ann. Phys. 303, 2 (2003).
${ }^{11}$ E. Dennis, A. Kitaev, A. Landahl, and J. Preskill, J. Math. Phys. 43, 4452 (2002).

${ }^{12}$ Z. Nussinov and G. Ortiz, Phys. Rev. B 76, 014530 (2007); arXiv:cond-mat/0702377 (unpublished).

${ }^{13}$ C. Castelnovo and C. Chamon, Phys. Rev. B 76, 184442 (2007); 78, 155120 (2008).

${ }^{14}$ R. Alicki, M. Fannes, and M. Horodecki, J. Phys. A: Math. Theor. 40, 6451 (2007); J. Phys. A: Math. Theor. 42, 065303 (2009).

${ }^{15}$ A. Kay and R. Colbeck, arXiv:0810.3557 (unpublished); S. Bravyi and B. Terhal, New J. Phys. 11, 043029 (2009).

${ }^{16}$ R. Alicki, M. Horodecki, P. Horodecki, and R. Horodecki, arXiv:0811.0033 (unpublished).

${ }^{17}$ S. Trebst, P. Werner, M. Troyer, K. Shtengel, and C. Nayak, Phys. Rev. Lett. 98, 070602 (2007); A. Hamma, W. Zhang, S. Haas, and D. A. Lidar, Phys. Rev. B 77, 155111 (2008).

${ }^{18}$ A. Hamma and D. A. Lidar, Phys. Rev. Lett. 100, 030502 (2008).

${ }^{19}$ G. D. Mahan, Many-Particle Physics, 2nd ed. (Plenum, New York, 1990).

${ }^{20}$ A. M. Salzberg, J. Math. Phys. 6, 158 (1965); P.-H. Chavanis, Eur. Phys. J. B 57, 391 (2007).

${ }^{21}$ T. Nakayama, K. Yakubo, and R. L. Orbach, Rev. Mod. Phys. 66, 381 (1994).

${ }^{22}$ X.-G. Wen (private communication). 